\title{
Analysis of cod-liver oil adulteration using Fourier Transform Infrared (FTIR) spectroscopy.
}

\begin{abstract}
Analysis of the adulteration of cod-liver oil with much cheaper oil-like animal fats has become attractive in recent years. This study highlights an application of Fourier transform infrared (FTIR) spectroscopy as a nondestructive and fast technique for the determination of adulterants in cod-liver oil. Attenuated total reflectance measurements were made on pure cod-liver oil and cod-liver oil adulterated with different concentrations of lard $(0.5-50 \% \mathrm{v} / \mathrm{v}$ in cod-liver oil). A chemometrics partial least squares (PLS) calibration model was developed for quantitative measurement of the adulterant. Discriminant analysis method was used to classify cod-liver oil samples from common animal fats (beef, chicken, mutton, and lard) based on their infrared spectra. Discriminant analysis carried out using seven principal components was able to classify the samples as pure or adulterated cod-liver oil based on their FTIR spectra at the selected fingerprint regions $(1,500-1,030 \mathrm{~cm}-1)$.
\end{abstract}

Keyword: Adulteration; Cod-liver oil; Lard; PLS; Discriminant analysis; FTIR; Spectroscopy; Lipid chemistry; Lipid analysis. 